\title{
Origin of the inner ring in photoluminescence patterns of quantum well excitons
}

\author{
A. L. Ivanov, ${ }^{1}$ L. E. Smallwood, ${ }^{1}$ A. T. Hammack, ${ }^{2}$ Sen Yang, ${ }^{2}$ L. V. Butov, ${ }^{2}$ and A. C. Gossard ${ }^{3}$ \\ ${ }^{1}$ Department of Physics and Astronomy, \\ Cardiff University, Cardiff CF24 3YB, United Kingdom \\ ${ }^{2}$ Department of Physics, University of California at San Diego, La Jolla, CA 92093-0319 \\ ${ }^{3}$ Materials Department, University of California at Santa Barbara, \\ Santa Barbara, California 93106-5050
}

(Dated: November 11, 2018)

\begin{abstract}
In order to explain and model the inner ring in photoluminescence (PL) patterns of indirect excitons in GaAs/AlGaAs quantum wells (QWs), we develop a microscopic approach formulated in terms of coupled nonlinear equations for the diffusion, thermalization and optical decay of the particles. The origin of the inner ring is unambiguously identified: it is due to cooling of indirect excitons in their propagation from the excitation spot. We infer that in our high-quality structures the in-plane diffusion coefficient $D_{\mathrm{x}}^{(2 \mathrm{~d})} \simeq$ $10-30 \mathrm{~cm}^{2} / \mathrm{s}$ and the amplitude of the disorder potential $U_{0} / 2 \simeq 0.45 \mathrm{meV}$.
\end{abstract}

PACS numbers: 73.63.Hs, 78.67.De, 05.30.Jp 
More than two decades ago, long-distance diffusion and drift transport of charge-neutral excitons with a long lifetime was optically visualized in bulk $\mathrm{Si}$ and $\mathrm{Cu}_{2} \mathrm{O}$ [1, 2]. In these earlier works, strain gradient potential traps were used to induce the drift motion. Due to the low particle concentrations, the transport was described in terms of a classical picture, with no quantum-statistical corrections, and the diffusion coefficient was attributed to exciton-phonon scattering. In-plane propagation of long-lived indirect excitons in coupled QWs over large distances has also been reported [3, 4, 5, 6, 7]: in this case one has a unique possibility to optically map the quasi-twodimensional motion of composite bosons. Furthermore, the density $n_{2 \mathrm{~d}}$ of indirect excitons can be large enough to ensure nonclassical population of the ground-energy state, $N_{E=0}=e^{T_{0} / T}-1 \gtrsim 1$, where $T_{0}=\left(\pi \hbar^{2} n_{2 \mathrm{~d}}\right) /\left(2 k_{\mathrm{B}} M_{\mathrm{x}}\right)$ and $T$ are the degeneracy temperature and exciton temperature, respectively, and $M_{\mathrm{x}}$ is the exciton in-plane translational mass (for a recent review of cold exciton gases in coupled QWs see Ref. [8]).

One of the most striking features of photoluminescence associated with indirect excitons is the appearance of two PL rings [6]. While the second, external ring has already been explained in terms of in-plane spatially separated electrons and holes [9, 10], the origin of the first, inner ring, which arises purely due to the transport of indirect excitons, remained unclear. The underlying physical picture we propose to explain this ring is that in the optically-pumped area the exciton temperature $T$ is much larger than the lattice temperature $T_{\mathrm{b}}$. As a result, the optical decay of excitons is suppressed, but while they diffuse out they cool down and eventually become opticallyactive, giving rise to a local increase of the PL signal. Thus the first ring is a generic feature of $\mathrm{cw}$ PL from excitons, direct or indirect, that are nonresonantly excited in high-quality QWs.

In this Letter we develop a microscopic theory for the long-range transport, thermalization and optical decay of QW excitons, model the inner PL ring, show an effective screening of QW disorder for $n_{2 \mathrm{~d}} \gtrsim 10^{10} \mathrm{~cm}^{-2}$, and analyze the quantum-statistical corrections. By numerically fitting the PL spectra we clarify the main scattering channels which contribute to the diffusion of indirect excitons and evaluate the diffusion coefficient and amplitude of the QW disorder potential. In the proposed model, the exciton temperature $T$ is affected by heating due to the optical excitation, heating due to the LA-phonon assisted conversion of the mean-field energy into the internal energy, and recombination heating or cooling due to the optical decay of low-energy excitons. The mean-field energy of indirect excitons also gives rise to a potential energy gradient and, therefore, to the in-plane drift motion.

In the experiments, the PL pattern is imaged by a nitrogen-cooled CCD camera with the spectral 
selection by an interference filter adjusted to the indirect exciton energy. Fine adjustment of the filtering energy is done by rotating two interference filters relative to the optical axis. As a result, we are able to remove the low-energy bulk emission that otherwise dominates the spectrum near the excitation spot [6], and observe a two-dimensional spatial image of the inner ring (see Figs. 1a, 1c, and 1d). Note that the inner PL ring can be missed if the bulk emission is not removed from the PL signal. In Figs. 1e and 1f we plot the measured exciton PL in the energy-coordinate plane. The exciton energy $E_{\mathrm{PL}}$ decreases with increasing distance from the excitation spot, as detailed below. This results in an arrow-shaped profile of the exciton PL images in the $E-x$ coordinates. The external ring is also seen at high excitations, both in $x-y$ (Fig. 1a) and $E$ - $x$ (Fig. 1e) coordinates. The excitation is done by a HeNe laser at $633 \mathrm{~nm}$ (the laser spot is a Gaussian with FWHM $=2 \sigma \simeq 6 \mu \mathrm{m}$, the excitation power $\left.P_{\mathrm{ex}}=1-400 \mu \mathrm{W}\right)$. The coupled QW structure with two $8 \mathrm{~nm}$ GaAs QWs separated by a $4 \mathrm{~nm} \mathrm{Al}_{0.33} \mathrm{Ga}_{0.67} \mathrm{As}$ barrier is grown by molecular beam epitaxy.

Our approach to the transport, relaxation and PL dynamics of indirect excitons is formulated in terms of three coupled nonlinear equations: a quantum diffusion equation for $n_{2 \mathrm{~d}}$, a thermalization equation for $T$, and an equation for the optical lifetime $\tau_{\mathrm{opt}}=1 / \Gamma_{\mathrm{opt}}$. The quantum-statistical corrections, which enhance the nonlinear effects, are included in the description.

The nonlinear quantum diffusion equation is given by

$$
\frac{\partial n_{2 \mathrm{~d}}}{\partial t}=\nabla\left[D_{\mathrm{x}}^{(2 \mathrm{~d})} \nabla n_{2 \mathrm{~d}}+\mu_{\mathrm{x}}^{(2 \mathrm{~d})} n_{2 \mathrm{~d}} \nabla\left(u_{0} n_{2 \mathrm{~d}}+U_{\mathrm{QW}}\right)\right]-\Gamma_{\mathrm{opt}} n_{2 \mathrm{~d}}+\Lambda,
$$

where $\Gamma_{\mathrm{opt}}, D_{\mathrm{x}}^{(2 \mathrm{~d})}, \mu_{\mathrm{x}}^{(2 \mathrm{~d})}$, and $\Lambda$ are the radiative decay rate, diffusion coefficient, mobility, and generation rate of $\mathrm{QW}$ excitons, respectively, $U_{\mathrm{QW}}=U_{\text {rand }}\left(\mathbf{r}_{\|}\right)$is a random potential due to the $\mathrm{QW}$ thickness and alloy fluctuations, and the operator $\nabla$ is defined in terms of the in-plane coordinate vector $\mathbf{r}_{\|}$. The generalized Einstein relationship [11], $\mu_{\mathrm{x}}^{(2 \mathrm{~d})}=D_{\mathrm{x}}^{(2 \mathrm{~d})}\left[\left(e^{T_{0} / T}-1\right) /\left(k_{\mathrm{B}} T_{0}\right)\right]$, which yields the classical limit $\mu_{\mathrm{x}}^{(2 \mathrm{~d})}=D_{\mathrm{x}}^{(2 \mathrm{~d})} /\left(k_{\mathrm{B}} T\right)$ for $T \gg T_{0}$, strongly enhances the nonlinearity of the diffusion Eq. (1) for $T \lesssim T_{0}$. The positive mean-field energy $u_{0} n_{2 \mathrm{~d}}$ on the right-hand side (r.h.s.) of Eq. (1) is due to the well-defined dipole-dipole repulsive interaction of indirect excitons [12, 13, 14]. Here $u_{0}=4 \pi\left(\mathrm{e}^{2} / \varepsilon_{\mathrm{b}}\right) d_{\mathrm{z}}, \varepsilon_{\mathrm{b}}$ is the background dielectric constant, and $d_{\mathrm{z}}$ is the separation between electron and hole layers. The mean-field energy gives rise to the in-plane drift motion with the velocity $\mathbf{v}_{\text {drift }}=-\mu_{\mathrm{x}}^{(2 \mathrm{~d})} u_{0} \nabla n_{2 \mathrm{~d}}$. As a result, an effective screening of the disorder potential $U_{\text {rand }}$ by dipole-dipole interacting indirect excitons builds up with increasing $n_{2 \mathrm{~d}}$ [11, 15]: the excitons tend to accumulate near the minima of $U_{\text {rand }}\left(\mathbf{r}_{\|}\right)$[local increase of $u_{0} n_{2 \mathrm{~d}}\left(\mathbf{r}_{\|}\right)$] and avoid the maxima of $U_{\text {rand }}\left(\mathbf{r}_{\|}\right)$[local decrease of $u_{0} n_{2 \mathrm{~d}}\left(\mathbf{r}_{\|}\right)$]. As we show below, in our high-quality structures 
$U^{(0)}=2\left\langle\left|U_{\text {rand }}\left(\mathbf{r}_{\|}\right)\right|\right\rangle \simeq 0.9 \mathrm{meV}$ and the mean-free energy $u_{0} n_{2 \mathrm{~d}}^{(0)} \simeq 1.6 \mathrm{meV}$ for $n_{2 \mathrm{~d}}^{(0)}=10^{10} \mathrm{~cm}^{-2}$, so that at low exciton temperatures $T \sim 1 \mathrm{~K}$ the $\mathrm{QW}$ disorder is strongly screened and practically removed for $n_{2 \mathrm{~d}} \gtrsim n_{2 \mathrm{~d}}^{(0)}$.

The temperature dynamics of excitons is given by

$$
\begin{aligned}
& \frac{\partial}{\partial t} T=\left(\frac{\partial T}{\partial t}\right)_{n_{2 \mathrm{~d}}}+\frac{S_{\mathrm{pump}}+S_{\mathrm{opt}}+S_{\mathrm{d}}}{2 k_{\mathrm{B}} T I_{1}-k_{\mathrm{B}} T_{0} I_{2}}, \\
&\left(\frac{\partial T}{\partial t}\right)_{n_{2 \mathrm{~d}}}=-\frac{2 \pi}{\tau_{\mathrm{sc}}}\left(\frac{T^{2}}{T_{0}}\right)\left(1-e^{-T_{0} / T}\right) \int_{1}^{\infty} d \varepsilon \varepsilon \sqrt{\frac{\varepsilon}{\varepsilon-1}} \\
& \quad \times \frac{\left|F_{z}(a \sqrt{\varepsilon(\varepsilon-1})\right|^{2}}{\left(e^{\varepsilon E_{0} / k_{\mathrm{B}} T_{\mathrm{b}}}-1\right)} \frac{e^{\varepsilon E_{0} / k_{\mathrm{B}} T_{\mathrm{b}}}-e^{\varepsilon E_{0} / k_{\mathrm{B}} T}}{\left(e^{\varepsilon E_{0} / k_{\mathrm{B}} T}+e^{-T_{0} / T}-1\right)},
\end{aligned}
$$

where $I_{1}=\left(1-e^{-T_{0} / T}\right) \int_{0}^{\infty} d z\left[z /\left(e^{z}+e^{-T_{0} / T}-1\right)\right], I_{2}=e^{-T_{0} / T} \int_{0}^{\infty} d z\left[\left(z e^{z}\right) /\left(e^{z}+e^{-T_{0} / T}-1\right)^{2}\right]$. Equation (3) describes the LA-phonon assisted thermalization of indirect excitons: $\tau_{\mathrm{sc}}=\left(\pi^{2} \hbar^{4} \rho\right) /\left(D_{\mathrm{dp}}^{2} M_{\mathrm{x}}^{3} v_{\mathrm{s}}\right)$ is the characteristic scattering time, $E_{0}=2 M_{\mathrm{x}} v_{\mathrm{s}}^{2}, v_{\mathrm{s}}$ is the longitudinal sound velocity, $\rho$ is the crystal density, and $D_{\mathrm{dp}}$ is the deformation potential of exciton - LA-phonon interaction. The formfactor $F_{z}(\chi)=[\sin (\chi) / \chi]\left[e^{i \chi} /\left(1-\chi^{2} / \pi^{2}\right)\right]$ refers to a QW confinement potential and characterizes a spectral band of bulk LA-phonons which interact with indirect excitons, $a=\left(d_{\mathrm{QW}} M_{\mathrm{x}} v_{\mathrm{s}}\right) / \hbar$, and $d_{\mathrm{QW}}$ is the QW thickness. For $1.5 \mathrm{~K} \lesssim T \lesssim 6.5 \mathrm{~K}$ and $n_{2 \mathrm{~d}}=10^{10} \mathrm{~cm}^{-2}$, relevant to our experiments, Eq. (3) yields a thermalization time $0.15 \mathrm{~ns} \gtrsim \tau_{\text {th }} \gtrsim 0.03 \mathrm{~ns}$. The strong increase of $\tau_{\text {th }}$ with decreasing $T$ is due to classical slowing down of the relaxation kinetics, which occurs at $k_{\mathrm{B}} T \sim E_{0}$ (excitons with energies $E \leq E_{0} / 4$ cannot emit LA-phonons) [16].

Heating of indirect excitons by the laser pulse is given by the term $S_{\text {pump }}=\left(E_{\text {inc }}-k_{\mathrm{B}} T I_{2}\right) \Lambda_{T_{0}}>0$ on the r.h.s. of Eq. (2). Here, $\Lambda_{T_{0}}=\left[\left(\pi \hbar^{2}\right) /\left(2 k_{\mathrm{B}} M_{\mathrm{x}}\right)\right] \Lambda\left(t, \mathbf{r}_{\|}\right)$. The generation of indirect excitons is a secondary process, mainly due to quantum tunnelling of photoexcited direct excitons to the energetically more favorable states of indirect excitons. The excess energy $E_{\text {inc }}$ of a created (incoming) indirect exciton is large: it exceeds the energy splitting between the direct and indirect excitons, which is about $20 \mathrm{meV}$. For the highest generation rates used in the experiments, the exciton temperature $T^{\max } \simeq 6.4 \mathrm{~K}$ at the laser spot centre is much larger than $T_{\mathrm{b}} \simeq 1.5 \mathrm{~K}$.

Recombination heating or cooling of QW excitons is given by the term $S_{\text {opt }}=\left[k_{\mathrm{B}} T I_{2} \Gamma_{\mathrm{opt}}-\right.$ $\left.E_{\gamma} \Gamma_{\text {opt }}^{\mathrm{E}}\right] T_{0}$ on the r.h.s. of Eq. (2), where

$$
\Gamma_{\mathrm{opt}}=\frac{1}{2 \tau_{\mathrm{R}}}\left(\frac{E_{\gamma}}{k_{\mathrm{B}} T_{0}}\right) \int_{0}^{1} \frac{1+z^{2}}{B e^{-z^{2} E_{\gamma} / k_{\mathrm{B}} T}-1} d z
$$

and $\Gamma_{\mathrm{opt}}^{\mathrm{E}}=\left[E_{\gamma} /\left(2 \tau_{\mathrm{R}} k_{\mathrm{B}} T_{0}\right)\right] \int_{0}^{1}\left[\left(1-z^{4}\right) /\left(B e^{-z^{2} E_{\gamma} / k_{\mathrm{B}} T}-1\right)\right] d z$ are the optical decay rates for the concentration and energy density of indirect excitons, respectively, $\tau_{\mathrm{R}}$ is the intrinsic radiative 


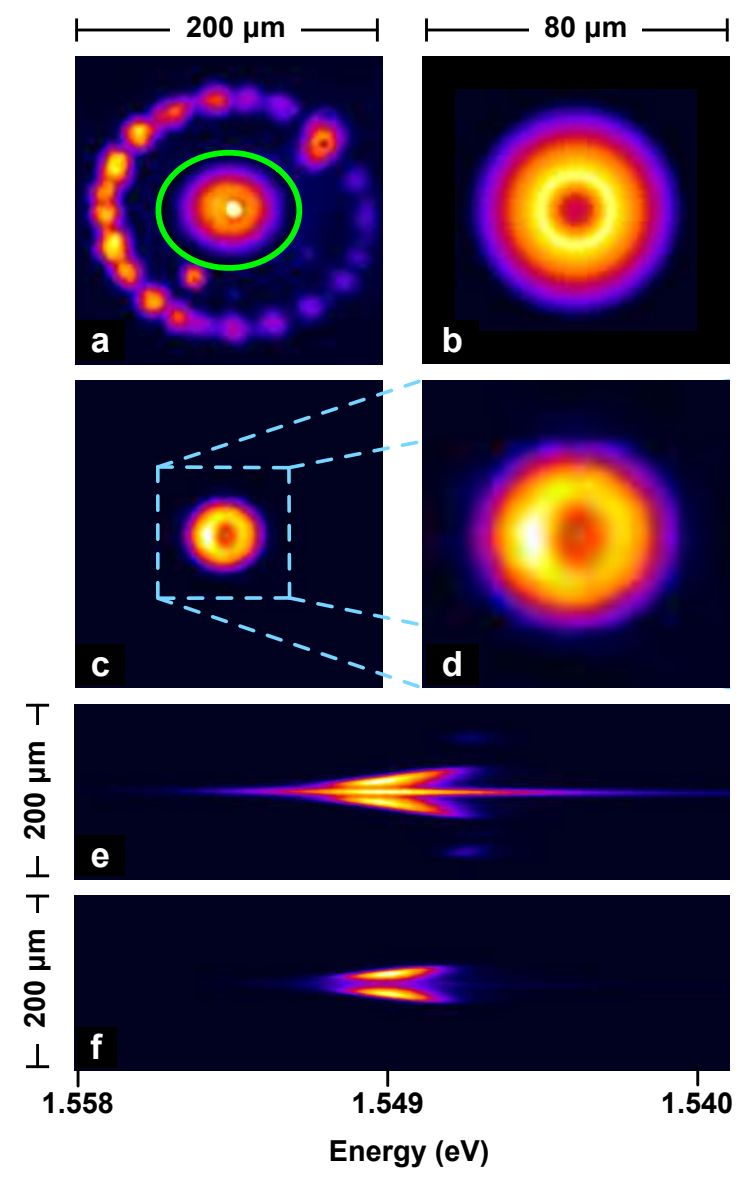

FIG. 1: (a), (c)-(f) Experimental and (b) calculated patterns of the PL signal from indirect excitons. In (a), the PL intensity in the area within the green circle is reduced by a constant factor for better visualization. A bright spot in the middle of the inner ring in (a) is due to residual bulk emission. (e) and (f) Image of the PL signal in the $E$ - $x$ coordinates. The external PL ring is also seen for high excitations, both in (a) $x-y$ and (e) $E$ - $x$ coordinates. For (a) and (e) the excitation power is $P_{\mathrm{ex}}=250 \mu \mathrm{W}$ and for (b)-(d) and (f) $P_{\mathrm{ex}}=75 \mu \mathrm{W}$, respectively, and $T_{\mathrm{b}}=1.5 \mathrm{~K}$.

lifetime of indirect excitons, $E_{\gamma}=p_{0}^{2} /\left(2 M_{\mathrm{x}}\right), B=\left(e^{E_{\gamma} / k_{\mathrm{B}} T}\right) /\left(1-e^{-T_{0} / T}\right)$, and $p_{0}=\left(E_{\mathrm{x}} \sqrt{\varepsilon_{\mathrm{b}}}\right) / c$ ( $E_{\mathrm{x}}$ is the total energy of a ground-state indirect exciton). In contrast with the evaporative cooling schemes used in atomic optics to remove high-energy atoms from magnetic traps [17, 18], the optical evaporation of QW excitons is an inherent process, which deals with the lowest-energy particles, $0 \leq E \leq E_{\gamma}$, from the radiative zone. Both signs of $S_{\mathrm{opt}}$ can be realized: $S_{\mathrm{opt}}>0$ $\left(S_{\text {opt }}<0\right)$, i.e., recombination heating (cooling) of indirect excitons for $k_{\mathrm{B}} T \gtrsim E_{\gamma}$ and $k_{\mathrm{B}} T_{0}$ $\left(k_{\mathrm{B}} T \ll E_{\gamma}\right.$ and $\left.k_{\mathrm{B}} T_{0}\right)$. In our case recombination heating occurs: far away from the excitation spot $T$ exceeds $T_{\mathrm{b}}$ by about $3 \mathrm{mK}$, i.e., due to relatively high $T_{\mathrm{b}}$, the effect is small. Note that an 

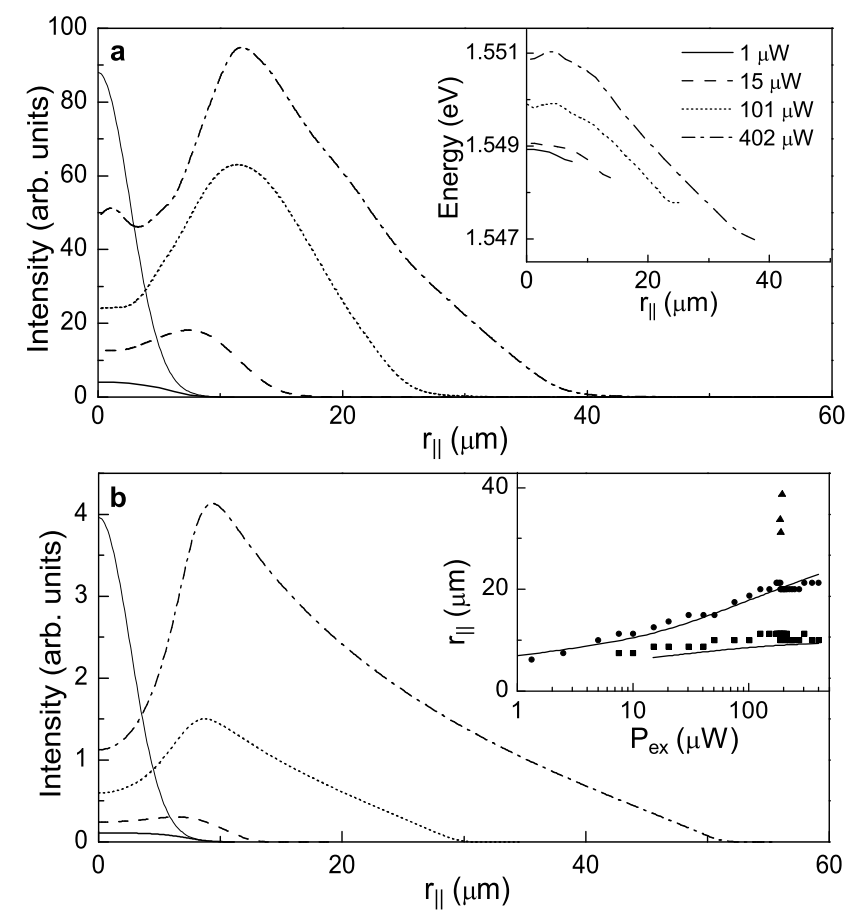

FIG. 2: The PL intensity $I_{\mathrm{PL}}$, (a) measured and (b) calculated with Eqs. (1)-(4), against radius $r_{\|}$for four optical excitation powers $P_{\mathrm{ex}}$. The Gaussian profile of the optical excitation, with $\sigma=3 \mu \mathrm{m}$, is shown by the thin solid lines. The cryostat temperature $T_{\mathrm{b}}=1.5 \mathrm{~K}$. In numerical evaluations the following parameters are used: $D_{\mathrm{dp}}=9.6 \mathrm{eV}, \tau_{\mathrm{R}}=13 \mathrm{~ns}, E_{\mathrm{inc}}=20 \mathrm{meV}, M_{\mathrm{x}}=0.215 m_{0}, d_{\mathrm{QW}}=8 \mathrm{~nm}$, and $d_{\mathrm{z}}=11.5 \mathrm{~nm}$. The best fitting parameters are $U_{0}=0.9 \mathrm{meV}, D_{\mathrm{x}-\mathrm{imp}}^{(2 d)}=60 \mathrm{~cm}^{2} / \mathrm{s}$, and $C_{\mathrm{x}-\mathrm{x}}=15 \mathrm{~cm}^{2} / \mathrm{s}$. The upper inset: the energy position of the PL line, $E_{\mathrm{PL}}=E_{\mathrm{PL}}\left(r_{\|}\right)$. The lower inset: the measured (square points) and calculated (solid line) inner ring radius $r_{\|}^{\mathrm{rg}}$ versus $P_{\mathrm{ex}}$. The triangular points refer to the external PL ring. The measured (circle points) and calculated (solid line) HWHM spatial extension of the PL signal against $r_{\|}$.

effective recombination cooling of particles takes place for $T_{\mathrm{b}} \lesssim 0.1 \mathrm{~K}$ : the effect is strong and reduces $\tau_{\text {th }}$ by $\gtrsim 20 \%$.

Heating of excitons by in-plane drift is given by $S_{\mathrm{d}}=-T_{0} u_{0}\left(\mathbf{v}_{\mathrm{tot}} \cdot \nabla n_{2 \mathrm{~d}}\right)$ on the r.h.s. of Eq. (2), where $\mathbf{v}_{\text {tot }}=\mathbf{v}_{\text {diff }}+\mathbf{v}_{\text {drift }}$ and $\mathbf{v}_{\text {diff }}=-\left(D_{\mathrm{x}}^{(2 \mathrm{~d})} / n_{2 \mathrm{~d}}\right) \nabla n_{2 \mathrm{~d}}$. This heating mechanism is due to conversion of the mean-field energy into the internal one. The effect is particularly important for $k_{\mathrm{B}} T \gg E_{0}$ $\left(E_{0} / k_{\mathrm{B}} \simeq 0.4 \mathrm{~K}\right)$, when the momentum relaxation time is much less than $\tau_{\text {th }}$.

In our model, the diffusion coefficient $D_{\mathrm{x}}^{(2 \mathrm{~d})}=\left(D_{\mathrm{x}-\mathrm{x}}^{(2 \mathrm{~d})} D_{\mathrm{x}-\mathrm{imp}}^{(2 \mathrm{~d})}\right) /\left(D_{\mathrm{x}-\mathrm{x}}^{(2 \mathrm{~d})}+D_{\mathrm{x}-\mathrm{imp}}^{(2 \mathrm{~d})}\right)$ has two contributions: diffusion due to scattering by imperfections (QW impurities and bulk LA-phonons), with $D_{\mathrm{x}-\mathrm{imp}}^{(2 \mathrm{~d})}=D_{\mathrm{x}-\mathrm{imp}}^{(2 \mathrm{~d})}\left(T_{\mathrm{b}}\right)$, and self-diffusion due to exciton-exciton scattering. The latter 
channel is important for $n_{2 \mathrm{~d}} \gtrsim 10^{10} \mathrm{~cm}^{-2}$ and $D_{\mathrm{x}-\mathrm{x}}^{(2 \mathrm{~d})}$ is approximated by $D_{\mathrm{x}-\mathrm{x}}^{(2 \mathrm{~d})}=C_{\mathrm{x}-\mathrm{x}}\left(T / T_{0}\right)$ [11]. For $r_{\|}$far away from the excitation spot, the asymptotic solution of Eqs. (11)-(4) yields $I_{\mathrm{PL}} \propto \exp \left[-\left(\Gamma_{\mathrm{opt}} / D_{\mathrm{x}-\mathrm{imp}}^{(2 \mathrm{~d})}\right)^{1 / 2} r_{\|}\right]$. In contrast, the experimental data show a much more steep decay of the PL signal and its spatial pinning at a critical radius $r_{\|}^{\mathrm{cr}}=r_{\|}^{\mathrm{cr}}\left(P_{\mathrm{ex}}\right)\left(\mathrm{e} . \mathrm{g}\right.$., $r_{\|}^{\mathrm{cr}} \simeq 40 \mu \mathrm{m}$ for $P_{\text {ex }}=402 \mu \mathrm{W}$, see Fig. $2 \mathrm{a}$ ). We attribute such a behaviour to the $n_{2 \mathrm{~d}}$-dependent screening of long-range-correlated QW disorder by dipole-dipole interacting indirect excitons. The narrowing effect is illustrated in Fig. 3a for a particular realization of the disorder potential $U_{\text {rand }}\left(r_{\|}\right)$. In order to include the long-range-correlated disorder we use a thermionic model [11] which operates with $n_{2 \mathrm{~d}^{-}}$and $T$-dependent diffusion coefficient $\tilde{D}_{\mathrm{x}}^{(2 \mathrm{~d})}=D_{\mathrm{x}}^{(2 \mathrm{~d})} \exp \left[-U^{(0)} /\left(k_{\mathrm{B}} T+u_{0} n_{2 \mathrm{~d}}\right)\right]$. Note that for $r_{\|} \simeq r_{\|}^{\text {cr }}$, where the narrowing effect is weak due to small $n_{2 \mathrm{~d}}$, a more adequate description of the diffusion and thermalization kinetics deals with phonon-assisted hopping between the QW localized states [19, 20].

In order to model the experimental results within the developed microscopic picture, we solve Eqs. (1)-(4) numerically for a stationary, cylindrically-symmetric optical excitation profile, so that the generation rate $\Lambda\left(\mathbf{r}_{\|}, t\right) \equiv \Lambda\left(r_{\|}\right) \propto P_{\mathrm{ex}} \exp \left(-r_{\|}^{2} / \sigma^{2}\right)$. The best fit for the experimental data plotted in Fig. 2a yields $U_{0}=0.9 \mathrm{meV}, D_{\mathrm{x}-\mathrm{imp}}^{(2 \mathrm{~d})}=60 \mathrm{~cm}^{2} / \mathrm{s}$, and $C_{\mathrm{x}-\mathrm{x}}=15 \mathrm{~cm}^{2} / \mathrm{s}$. The calculated spatial profile of the PL signal, $I_{\mathrm{PL}}=I_{\mathrm{PL}}\left(r_{\|}\right)$, is shown in Fig. $2 \mathrm{~b}$ for various pump powers $P_{\mathrm{ex}}$. While the density profile $n_{2 \mathrm{~d}}=n_{2 \mathrm{~d}}\left(r_{\|}\right)$always has a bell-like shape (see Fig. $3 \mathrm{~b}$ and the inset of Fig. 2a), with increasing $P_{\mathrm{ex}}$ the inner PL ring develops in the $I_{\mathrm{PL}}$-profile. This is in a complete agreement with the observations. The inner ring has a nearly classical origin, and arises due to heating of indirect excitons by the optical excitation $\left[S_{\text {pump }}\right.$ term in Eq. (2)]: with increasing $r_{\|}$the exciton temperature $T$ rapidly decreases towards $T_{\mathrm{b}}$ (see Fig. 3b); as a result, the optical lifetime $\tau_{\mathrm{opt}}=1 / \Gamma_{\mathrm{opt}}$ decreases too (see the inset of Fig. $3 \mathrm{~b}$ ), giving rise to a local increase of $I_{\mathrm{PL}}\left(r_{\|}\right)$at $r_{\|}=r_{\|}^{\mathrm{rg}}$. Thus the inner ring is a spatial counterpart of the PL-jump observed in the time-resolved experiments [21]. Our numerical simulations also reproduce the observed increase of $r_{\|}^{\mathrm{rg}}$ and the spatial extension of the PL area (HWHM of the signal) with increasing $P_{\mathrm{ex}}$ (see the inset of Fig. 2b).

The finding of the fitting parameters, which refer to the total diffusion coefficient $\tilde{D}_{\mathrm{x}}^{(2 \mathrm{~d})}$, is complex, i.e., we fit all the curves plotted in Fig. $2 \mathrm{a}\left[I_{\mathrm{PL}}=I_{\mathrm{PL}}\left(r_{\|}\right)\right.$and $E_{\mathrm{PL}}=E_{\mathrm{PL}}\left(r_{\|}\right)$for various $\left.P_{\text {ex }}\right]$ by using the same values of $U_{0}, D_{\mathrm{x}-\mathrm{imp}}^{(2 \mathrm{~d})}$, and $C_{\mathrm{x}-\mathrm{x}}$. The blue shift $\delta_{\mathrm{PL}} \geq 0$ of the PL energy $E_{\mathrm{PL}}$ is due to the mean-field interaction energy of indirect excitons, $\delta_{\mathrm{PL}}=E_{\mathrm{PL}}-E_{\mathrm{x}}=u_{0} n_{2 \mathrm{~d}}$ (see Figs. 1e-1f and the inset of Fig. 2a). Thus we use the measured $\delta_{\mathrm{PL}}$ to estimate $n_{2 \mathrm{~d}}^{\max }\left(r_{\|}=0\right)$, and 

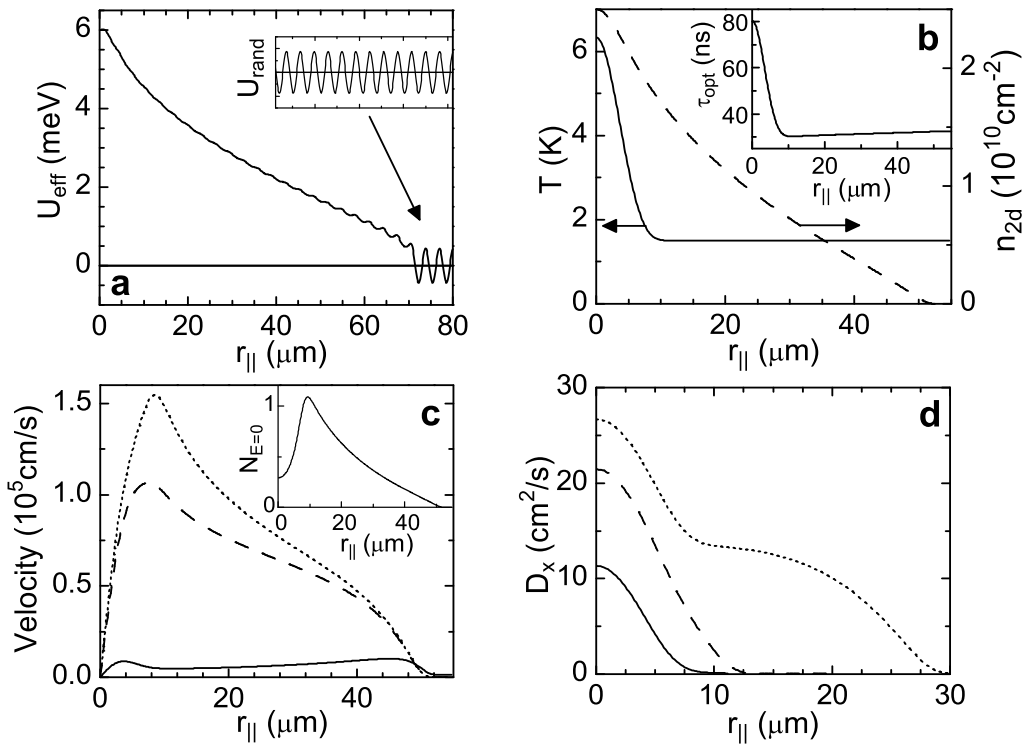

FIG. 3: (a) The narrowing effect: screening of the long-range-correlated disorder potential $U_{\text {rand }}\left(\mathbf{r}_{\|}\right)$by dipole-dipole interacting indirect excitons. The QW effective potential $U_{\text {eff }}\left(r_{\|}\right)=U_{\text {rand }}\left(r_{\|}\right)+u_{0} n_{2 \mathrm{~d}}\left(r_{\|}\right)$ calculated with Eqs. (1)-(4) for harmonic $U_{\text {rand }}\left(r_{\|}\right)$with $U_{0} / 2=0.45 \mathrm{meV}$ (see the inset). (b) $T=T\left(r_{\|}\right)$ (solid line) and $n_{2 \mathrm{~d}}=n_{2 \mathrm{~d}}\left(r_{\|}\right)$(dashed line). Inset: $\tau_{\mathrm{opt}}=\tau_{\mathrm{opt}}\left(r_{\|}\right)$. (c) Diffusion velocity $v_{\mathrm{diff}}=v_{\mathrm{diff}}\left(r_{\|}\right)$(solid line) and drift velocity $v_{\text {drift }}=v_{\text {drift }}\left(r_{\|}\right)$without (dashed line) and with (dotted line) quantum-statistical corrections. Inset: $N_{E=0}=N_{E=0}\left(r_{\|}\right)$. Plots (a)-(c) refer to the maximum excitation, $n_{2 \mathrm{~d}}^{\max }=2.5 \times 10^{10} \mathrm{~cm}^{-2}$ (see the solid lines in Fig. 2). (d) Diffusion coefficient $D_{\mathrm{x}}^{(2 \mathrm{~d})}=D_{\mathrm{x}}^{(2 \mathrm{~d})}\left(r_{\|}\right)$for $n_{2 \mathrm{~d}}^{\max }=0.16 \times 10^{10} \mathrm{~cm}^{-2}$ (solid line), $0.44 \times 10^{10} \mathrm{~cm}^{-2}$ (dashed line), and $1.27 \times 10^{10} \mathrm{~cm}^{-2}$ (dotted line). These values of $n_{2 \mathrm{~d}}^{\max }$ correspond to the PL signal shown in Fig. 2.

therefore the generation rate $\Lambda\left(r_{\|}=0\right)$, necessary for numerical modelling with Eqs. (11)-(4). The amplitude of the disorder potential $U_{0}$ determines the steepness of $I_{\mathrm{PL}}\left(r_{\|}>r_{\|}^{\mathrm{rg}}\right)$ and dependence $r_{\|}^{\mathrm{cr}}=r_{\|}^{\mathrm{cr}}\left(P_{\mathrm{ex}}\right)$ of the PL pinning radius. In turn, $D_{\mathrm{x}-\mathrm{imp}}^{(2 d)}$ and $C_{\mathrm{x}-\mathrm{x}}$ determine the ring contrast and $r_{\|}^{\mathrm{rg}}=r_{\|}^{\mathrm{rg}}\left(P_{\mathrm{ex}}\right)$ dependence. The total diffusion coefficient $\tilde{D}_{\mathrm{x}}^{(2 \mathrm{~d})}=\tilde{D}_{\mathrm{x}}^{(2 \mathrm{~d})}\left(r_{\|}\right)$is plotted in Fig. $3 \mathrm{~d}$.

In Fig. 3c we show that for the small size excitation spot $(\sigma \simeq 3 \mu \mathrm{m})$ used in the experiments, the drift velocity $v_{\text {drift }}$, due to the gradient of the mean-field interaction energy $u_{0} n_{2 \mathrm{~d}}\left(r_{\|}\right)$, is much larger than the diffusion velocity $v_{\text {diff }}$. The total velocity has a maximum value $v_{\text {tot }}^{\max }\left(r_{\|} \simeq r_{\|}^{\mathrm{rg}}\right) \simeq$ $1.5 \times 10^{5} \mathrm{~cm} / \mathrm{s}$ for $n_{2 \mathrm{~d}}^{\max }\left(r_{\|}=0\right) \simeq 2.5 \times 10^{10} \mathrm{~cm}^{-2}$ (see Fig. $3 \mathrm{c}$ ). Note that in our case the meanfield energy gradient $u_{0}\left|\nabla n_{2 \mathrm{~d}}\left(r_{\|} \simeq r_{\|}^{\mathrm{rg}}\right)\right| \simeq 1.6 \mathrm{eV} / \mathrm{cm}$ exceeds the maximum strain-induced gradient $|\nabla U| \simeq 0.4 \mathrm{eV} / \mathrm{cm}$ used in the experiments [1, 2]. 
Finally, we emphasize that in our experiments, which deal with the cryostat temperature $T_{\mathrm{b}}=$ $1.5 \mathrm{~K}$, nonclassical occupation numbers of modest values, $N_{E=0}^{\max }\left(r_{\|} \simeq r_{\|}^{\mathrm{rg}}\right) \simeq 1$, build up at the position of the inner ring (see the inset of Fig. 3c). As illustrated in Fig. 3c, in this case the quantum statistical corrections, e.g., to $v_{\text {diff }}$ and to the Einstein relationship, are about $35 \%$ and therefore cannot be neglected. Nonclassical statistics occurs at the position of the inner ring, where the exciton gas is already cold but still dense. Furthermore, for $T_{\mathrm{b}} \sim 0.1 \mathrm{~K}$ (not yet realized in an optical imaging experiment) numerical modelling with Eqs. (1)-(4) gives well-developed BoseEinstein statistics with $N_{E=0}^{\max }\left(r_{\|} \simeq r_{\|}^{\mathrm{rg}}\right) \gg 1$.

We appreciate valuable discussions with L. V. Keldysh, L. S. Levitov, L. Mouchliadis, and B. D. Simons. Support of this work by EU RTN Project HPRN-2002-00298 is gratefully acknowledged.

[1] M. A. Tamor and J. P. Wolfe, Phys. Rev. Lett. 44, 1703 (1980).

[2] D. P. Trauernicht, J. P. Wolfe, and A. Mysyrowicz, Phys. Rev. Lett. 52, 855 (1984).

[3] M. Hagn, A. Zrenner, G. Böhm, and G. Weimann, Appl. Phys. Lett. 67, 232 (1995).

[4] V. Negoita, D. W. Snoke, and K. Eberl, Phys. Rev. B 60 2661, (1999).

[5] A. V. Larionov, V. B. Timofeev, J. Hvam, and K. Soerensen, JETP 90, 1093 (2000) [Zh. Eksp. Teor. Fiz. 117, 1255 (2000)].

[6] L. V. Butov, A. C. Gossard, and D. S. Chemla, Nature 418, 751 (2002).

[7] Z. Vörös, R. Balili, D. W. Snoke, L. Pfeiffer, and K. West, Phys. Rev. Lett. 94, 226401 (2005).

[8] L. V. Butov, J. Phys.: Cond. Matter 16, R1577 (2004).

[9] L. V. Butov, L. S. Levitov, A. V. Mintsev, B. D. Simons, A. C. Gossard, and D. S. Chemla, Phys. Rev. Lett. 92, 117404 (2004).

[10] R. Rapaport, G. Chen, D. Snoke, S. H. Simon, L. Pfeiffer, K. West, Y. Liu, and S. Denev, Phys. Rev. Lett. 92, 117405 (2004).

[11] A. L. Ivanov, Europhys. Lett. 59, 586 (2002).

[12] D. Yoshioka and A. H. MacDonald, J. Phys. Soc. Jpn. 59, 4211 (1990).

[13] L. V. Butov, A. Zrenner, G. Abstreiter, G. Bohm, and G. Weimann, Phys. Rev. Lett. 73, 304 (1994).

[14] X. Zhu, P. B. Littlewood, M. Hybertsen, and T. M. Rice, Phys. Rev. Lett. 74, 1633 (1995).

[15] R. Zimmermann, Solid State Commun. 134, 43 (2005).

[16] A. L. Ivanov, P. B. Littlewood, and H. Haug, Phys. Rev. B 59, 5032 (1999); A. V. Soroko and A. L. 
Ivanov, Phys. Rev. B 65, 165310 (2002).

[17] E. A. Cornell and C. E. Wieman, Rev. Mod. Phys. 74, 875 (2002).

[18] W. Ketterle, Rev. Mod. Phys. 74, 1131 (2002).

[19] S. D. Baranovskii, R. Eichmann, and P. Thomas, Phys. Rev. B 58, 13081 (1998).

[20] M. Grassi Alessi, F. Fragano, A. Patanè, M. Capizzi, E. Runge, and R. Zimmermann, Phys. Rev. B 61, 10985 (2000).

[21] L. V. Butov, A. L. Ivanov, A. Imamoglu, P. B. Littlewood, A. A. Shashkin, V. T. Dolgopolov, K. L. Campman, and A. C. Gossard, Phys. Rev. Lett. 86, 5608 (2001). 